\title{
Accuracy of intraocular lens calculations based on fellow-eye biometry for phacovitrectomy for macula-off rhegmatogenous retinal detachments
}

\author{
Abdul R. El-Khayat $\mathbb{D}^{1} \cdot$ Alex J. Brent $\mathbb{D}^{1} \cdot$ Shamfa A. M. Peart ${ }^{1} \cdot$ Partha R. Chaudhuri $^{1}$ \\ Received: 3 November 2018 / Revised: 27 March 2019 / Accepted: 16 May 2019 / Published online: 10 June 2019 \\ (c) The Author(s), under exclusive licence to The Royal College of Ophthalmologists 2019
}

\begin{abstract}
Objectives To determine the accuracy of using fellow-eye biometry for intraocular lens calculations for phacovitrectomy for macula off rhegmatogenous retinal detachments.

Methods Retrospective case review of phacovitrectomies for consecutive macula off retinal detachments over 10 years. Optical and/or ultrasound biometry was performed for affected and fellow eyes. Prediction error was determined by calculating the difference between predicted and actual refractive outcomes. Results from fellow- and same-eye biometry were compared.

Results Forty-two eyes were included. The mean prediction errors for fellow- and same-eye biometry were $-0.01 \pm 1.09$ and $-1.22 \pm 2.32$ dioptres, respectively, indicating a myopic shift for same eye biometry calculations. The mean absolute prediction errors for fellow and same eye biometry were $0.73 \pm 0.80$ and $1.57 \pm 2.08$ dioptres, respectively. The difference was statistically significant $(P=0.016)$.

Conclusions When appropriate, intraocular lens calculations using fellow-eye biometry for phacovitrectomy for macula off rhegmatogenous retinal detachments are accurate and better than those from same-eye biometry.
\end{abstract}

\section{Introduction}

Visual rehabilitation after pars plana vitrectomy (PPV) may be impaired due to coexisting cataracts [1] or the frequent formation of cataracts after surgery [2]. Within 1 year of vitrectomy for retinal detachment, the majority of phakic eyes undergo cataract surgery [3]. Consequently, combined phacoemulsification and PPV (Phacovitrectomy) has become a common procedure, spurred on by advances in cataract and vitrectomy surgery [4]. It is cost-effective and allows for faster visual rehabilitation from a single recovery period [5]. PPV in aphakia or pseudophakia also allows for

Presented as an oral presentation at the British and Eire Association of Vitreoretinal Surgeons (BEAVRS) Annual Meeting, November $8-9,2018$.

Abdul R. El-Khayat

a_elkhayat@hotmail.com

1 Department of Ophthalmology, University Hospitals of Leicester NHS Trust, Leicestershire LE1 5WW, UK improved access to, and visualisation of, the peripheral retina [6], facilitated by the removal of lens opacities and the elimination of risk of lens touch.

Measurement of axial length (AL) is considered to be the most crucial step in intraocular lens (IOL) power calculation accuracy [7]. With retinal detachment, however, especially where the macula is off, AL measurements tend to be less accurate and underestimated [8-10]. Furthermore, AL measurements correlate with retinal detachment height [11], meaning that the level of error may be difficult to predict due to the variable height and dynamic nature of rhegmatogenous retinal detachments (RRDs) [10]. Multiple methods are in use for determining the AL in macula off (mac off) RRD, including A-scan and optical biometry for same and fellow eyes, manually adjusted biometry and delayed cataract surgery $[5,8,9,11]$. Currently there is no consensus on the optimal method $[5,8,9]$; however, since there is an inherent error in same-eye biometry, and individuals have little difference in $\mathrm{AL}$ between their eyes $(82.5 \%$ have $\leq 0.3 \mathrm{~mm}$ difference between eyes) [12], there is a strong argument for using fellow-eye biometry.

We conducted a retrospective study, looking at the accuracy of IOL calculations using fellow-eye biometry for 
phacovitrectomy for macula off RRD. For the same population, we also compared what the outcomes would have been for same-eye biometry.

\section{Methods}

This was a retrospective study of all consecutive macula off RRD patients who had phacovitrectomy by one surgeon (PRC) at Leicester Royal Infirmary, UK, from December 2007 to 2017. Inclusion criteria were a diagnosis of mac off RRD, phakia and available biometry, and post-op refraction. Exclusion criteria were failed RD surgery and poor visual outcome that precluded subjective refraction.

Axial lengths were measured by trained operators using optical biometry (OB) (IOL Master, Carl Zeis, Meditec, Jana, Germany). Only measurements with a signal to noise ratio (SNR) above 2 were included. A-scan ultrasound biometry (UB) (Accutome A-Scan Plus, Accutome Inc., Malvern, USA) was used when OB was not possible. IOL power was calculated using the SRK/T formula. An optimised A constant based on the User Group for Laser Interference Biometry data was used [13]. The target refractive outcome was either emmetropia or isometropia based on a discussion with patients and fellow-eye refraction. Fellow-eye OB was used for all patients where this was available. If this was not available then fellow-eye UB was used. The fellow-eye OB used for calculations included all readings from the fellow eye, including $\mathrm{K}$ readings.

Phacoemulsification was performed through a $2.2-\mathrm{mm}$, superior, clear corneal incision. A one-piece hydrophobic acrylic lens was injected into the capsular bag. Three port $23 \mathrm{G} \mathrm{PPV}$ was performed and sub-retinal fluid was aspirated with the assistance of heavy liquid. Retinopexy was performed using endo-photocoagulation. Endotamponade was achieved using either gas (SF6, C2F6) or silicone oil. Optometrists, either in-house or in the community, performed postoperative (postop) refraction at least 3 months after surgery or silicone oil removal. Only patients who achieved anatomical success and who had postop refraction were included in the study.

For all patients, the predicted postop spherical equivalent was determined by the IOL selected from the biometry printout. This would normally be the outcome closest to emmetropia or isometropia. Actual postop spherical equivalent was determined from refractions of patients at 3 months or later, and was used for fellow- and same-eye calculations. The accuracy of IOL calculations based on fellow-eye biometry was calculated by subtracting the actual postop spherical equivalent from the predicted postop spherical equivalent to give a prediction error. Mean absolute prediction error (MAE) was used as the primary outcome as opposed to the mean prediction error (ME), as ME may give a false representation of the error due to the averaging of positive and negative values. ME was still calculated, however, to determine whether on average patients had a myopic shift in their refractive outcome. Secondary outcomes included the percentage of patients who achieved an absolute prediction error within 0.5 dioptres (D), $1.00 \mathrm{D}, 2.00 \mathrm{D}$ and $>2.00 \mathrm{D}$. These data was compared with prediction errors if same-eye biometry had been used. The unpaired $t$-test (two-tailed) was used to determine the significance of the difference in outcomes between same- and fellow-eye biometry.

Further analyses were carried out looking at SNR and preop anisometropia. The SNR was compared between same- and fellow-eye biometry to see if there was a significant difference between the two, and to see if there was an inverse correlation between the SNR and the error in refractive outcome (absolute prediction error) for same eye biometry. Preop anisometropia was compared with absolute prediction error to see if this correlated with refractive surprise. Correlations were determined using the Pearson Correlation Coefficient $(r)$.

\section{Results}

Sixty-one cases were identified as having a phacovitrectomy for Mac off RRD over the 10-year period. After excluding those with insufficient data (five excluded), failed surgery (five excluded) and unavailable refraction (nine excluded), 42 cases were included in the analysis. Each patient was included once and for a single eye. Patients had a mean age of 60.1 years, $23(55 \%)$ were males and $38(90.5 \%)$ had OB. 17 patients had a target outcome of isometropia, $25 \mathrm{had}$ a target of emmetropia. Baseline characteristics of patients are summarised in Table 1.

\section{Fellow-eye biometry}

The ME for fellow-eye biometry was $-0.01 \mathrm{D}$ (SD 1.09 D) and ranged from $-2.05 \mathrm{D}$ to $+2.84 \mathrm{D}$. The MAE was 0.73 D (SD 0.80 D). In all, 23 (54.8\%) eyes achieved an absolute prediction error within $0.5 \mathrm{D}, 30(71.4 \%)$ within $1.0 \mathrm{D}, 36$ $(85.7 \%)$ within $2.0 \mathrm{D}$ and $6(14.3 \%)$ over $2.0 \mathrm{D}$.

\section{Same-eye biometry}

If same-eye biometry had been used, the ME would have been $-1.22 \mathrm{D}(\mathrm{SD} 2.32 \mathrm{D})$, ranging from $-11.54 \mathrm{D}$ to 2.54 D. The MAE would have been $1.57 \mathrm{D}$ (SD 2.08 D). Fourteen $(34.1 \%)$ eyes would have achieved an absolute prediction error within $0.5 \mathrm{D}, 24(58.5 \%)$ within $1.0 \mathrm{D}, 30$ (73.2\%) within $2.0 \mathrm{D}$ and $11(26.8 \%)$ over $2.0 \mathrm{D}$. Results for outcomes within $0.5 \mathrm{D}, 1.0 \mathrm{D}, 2.0 \mathrm{D}$ and $>2.0 \mathrm{D}$ are 
Table 1 Baseline characteristics of patients

\begin{tabular}{|c|c|}
\hline & Tot: \\
\hline \multicolumn{2}{|l|}{ AL measurement method } \\
\hline OB & 38 \\
\hline UB & 4 \\
\hline \multicolumn{2}{|l|}{ Tamponade used } \\
\hline SF6 & 15 \\
\hline $\mathrm{C} 2 \mathrm{~F} 6$ & 22 \\
\hline Silicone oil & 5 \\
\hline \multicolumn{2}{|l|}{ Comoorbidities } \\
\hline Amblyopia (same or fellow eye) & 3 \\
\hline AMD & 2 \\
\hline PVR & 2 \\
\hline Laser refractive surgery & 2 \\
\hline \multicolumn{2}{|l|}{ Detachment configuration } \\
\hline Total/sub-total & 6 \\
\hline$>/=6$ clock hours & 13 \\
\hline$<6$ clock hours & 23 \\
\hline \multicolumn{2}{|l|}{ Gender } \\
\hline Male & 23 \\
\hline Female & 19 \\
\hline \multicolumn{2}{|l|}{ IOL used } \\
\hline AcrySof SA60AT & 1 \\
\hline AcrySof SN60WF & 32 \\
\hline AcrySof MA60AC & 1 \\
\hline Hoya iSert 250 & 8 \\
\hline \multicolumn{2}{|l|}{ IOL formula } \\
\hline $\mathrm{SRK} / \mathrm{T}$ & 39 \\
\hline Haigis-L myopic & 2 \\
\hline Hoffer Q & 1 \\
\hline
\end{tabular}

$A L$ axial length, $O B$ optical biometry, $U B$ ultrasound biometry (A-scan), SF6 sulphur hexafluoride, $C 2 F 6$ perfluoroethane, $A M D$ age related macular degeneration, $P V R$ proliferative vitreoretinopathy

summarised in Fig. 1. The mean AL for same eye biometry was $0.52 \mathrm{~mm}$ shorter than that of fellow-eye biometry. This may have contributed to the myopic ME and the greater MAE of same eye outcomes.

The difference in MAE between fellow- and same-eye biometry $(0.84)$ was significant $(P=0.016)$. The results from same-eye biometry may have been skewed from an outlier ( -11.54 prediction error) caused by a very short $\mathrm{AL}$ measurement $(19.81 \mathrm{~mm})$. If this is excluded, same-eye biometry outcomes would be ME -0.96 (SD 1.65), MAE 1.33 (SD 1.36), and a difference in MAE of 0.60 $(P=0.017)$.

Since only 4 cases used UB, a subgroup analysis was performed for OB only, giving a MAE of 0.75 (SD 0.83) for fellow-eye and 1.58 (SD 2.11) for same-eye biometry, with a difference of $0.83(P=0.027)$. As shown, the difference in our primary outcome (MAE) between fellow-eye and

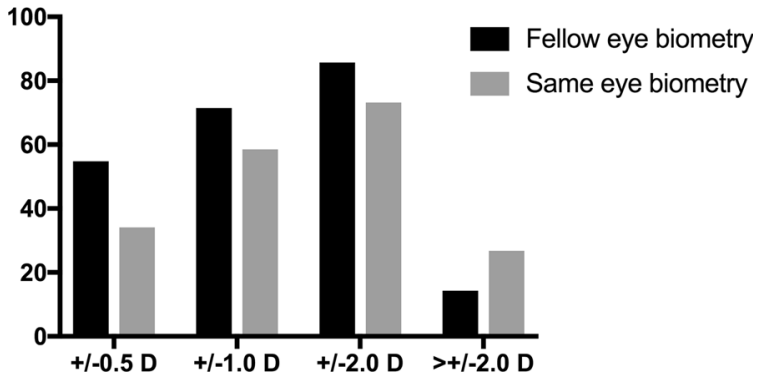

Fig. 1 Percentage of eyes achieving the refractive aim

same-eye biometry remained statistically significant when outliers and OB were excluded. Results are summarised in Table 2.

The SNR for same eye OB was lower than fellow eye OB in $80.6 \%$ of cases. However, there was no significant difference in mean SNR between same and fellow eyes $(P=0.505)$. An inverse correlation was found between the SNR and the absolute error for same eye biometry $(r=$ -0.247 ), however, this was not significant ( $P=0.152,95 \%$ CI -0.536-0.094).

There was no significant correlation between preop anisometropia and absolute prediction error using fellow-eye biometry $(r=0.147, P=0.415,95 \% \mathrm{CI}-0.207-0.467)$.

\section{Discussion}

To the best of our knowledge, this study is the only analysis of refractive outcomes using only fellow-eye biometry for phacovitrectomy for mac off RRD. Considering that combined phacovitrectomy is a common procedure $(10.7 \%$ of all vitrectomies for retinal detachment) [3] and that some surgeons advocate the routine use of fellow-eye biometry for all mac off RRDs [11], results for this biometry method are particularly pertinent.

Our results suggest that using fellow-eye biometry for IOL calculations for mac off RRD is accurate (MAE 0.73 D) and, as expected, does not lead to a myopic shift (ME -0.01). The vast majority of patients $(71.4 \%)$ were within $1.0 \mathrm{D}$ of their predicted refractive outcome, which is not far off some targets for routine cataract surgery [14].

Comparing these figures with refractive outcomes we would have achieved from same-eye biometry, calculations have a myopic shift (ME -1.22 D), are far more variable (range $14.1 \mathrm{D}$ vs $4.9 \mathrm{D}$, SD $2.32 \mathrm{D}$ vs $1.09 \mathrm{D}$ ) and are significantly less accurate (MAE $1.57 \mathrm{D}$ vs $0.73 \mathrm{D}(P=$ 0.016)). Furthermore, far fewer patients achieve an outcome within $1.0 \mathrm{D}$ of prediction (58.5\%).

Few studies, with varying biometry methods, have looked at refractive outcomes for phacovitrectomy for mac off RRD. Their results are summarised in Table 3. Drawing parallels is difficult as the studies often combined multiple 
Table 2 Summary of results (all outcomes in dioptres)

\begin{tabular}{|c|c|c|c|c|}
\hline & $\mathrm{ME}(\mathrm{SD})$ & Range & MAE (SD) & Difference in MAE \\
\hline All data & & & & $0.86(P=0.016)$ \\
\hline Fellow eye & $-0.01(1.09)$ & $-2.05-2.84$ & $0.73(0.80)$ & \\
\hline Same eye & $-1.22(2.32)$ & $-11.54-2.54$ & $1.58(2.08)$ & \\
\hline Excluding outlier & & & & $0.60(P=0.017)$ \\
\hline Fellow eye & $-0.01(1.09)$ & $-2.05-2.84$ & $0.73(0.80)$ & \\
\hline Same eye & $-0.96(1.65)$ & $-5.47-2.54$ & $1.33(1.36)$ & \\
\hline Excluding UB & & & & $0.83(P=0.027)$ \\
\hline Fellow eye & $0.04(1.12)$ & $-2.05-2.84$ & $0.75(0.83)$ & \\
\hline Same eye & $-1.20(2.35)$ & $-11.54-2.54$ & $1.58(2.11)$ & \\
\hline \multicolumn{5}{|c|}{$\begin{array}{l}M E \text { mean prediction error, } M A E \text { mean absolute prediction error, } U B \text { ultrasound biometry (A-scan), } P \text { value } \\
\text { using the unpaired two-tailed } t \text {-test }\end{array}$} \\
\hline Study & $\begin{array}{l}\text { Number } \\
\text { (macula off) }\end{array}$ & Biometry method & Same/fellow eye & $\begin{array}{l}\mathrm{MAE} \pm \mathrm{SD} \\
\text { (dioptres) }\end{array}$ \\
\hline \multirow{2}{*}{$\begin{array}{l}\text { El-Khayat et al. } \\
\text { (this study) }\end{array}$} & 42 & $\mathrm{OB}(38)+\mathrm{UB}(4)$ & Fellow eye & $0.73 \pm 0.80$ \\
\hline & 42 & $\mathrm{OB}(38)+\mathrm{UB}(4)$ & Same eye & $1.57 \pm 2.08$ \\
\hline \multirow{3}{*}{$\begin{array}{l}\text { Abou-Shousha et al. } \\
\text { [9] }\end{array}$} & 100 & OB & Same eye & $1.18 \pm 0.84$ \\
\hline & 100 & UB & Same eye & $1.33 \pm 1.06$ \\
\hline & 100 & Vector A/B scan & Same eye & $0.59 \pm 0.48$ \\
\hline \multirow[t]{3}{*}{ Rahman et al. [8] } & 54 & $\mathrm{OB}+\mathrm{UB}$ & $\begin{array}{l}\text { Same }(46)+ \\
\text { Fellow eye }(8)\end{array}$ & $0.73 \pm 0.71$ \\
\hline & 13 & OB & Same eye & $0.65 \pm 0.71$ \\
\hline & 33 & UB & Same eye & $0.77 \pm 0.72$ \\
\hline \multirow[t]{2}{*}{ Kim et al. [11] } & $\begin{array}{l}26 / 38(68 \%) \\
\text { mac off }\end{array}$ & Average UB + OB & Same eye & $0.81 \pm 0.81$ \\
\hline & $\begin{array}{l}14 / 25(56 \%) \\
\text { mac off }\end{array}$ & $\begin{array}{l}\text { Average } \mathrm{UB}+\mathrm{OB} \\
\text { delayed phaco }\end{array}$ & Same eye & $0.48 \pm 0.29$ \\
\hline
\end{tabular}

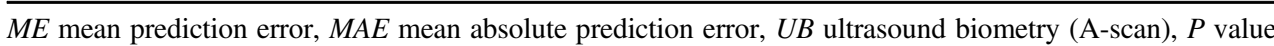
using the unpaired two-tailed $t$-test

\begin{tabular}{|c|c|c|c|c|}
\hline & $\mathrm{ME}(\mathrm{SD})$ & Range & MAE (SD) & Difference in MAE \\
\hline All data & & & & $0.86(P=0.016)$ \\
\hline Fellow eye & $-0.01(1.09)$ & $-2.05-2.84$ & $0.73(0.80)$ & \\
\hline Same eye & $-1.22(2.32)$ & $-11.54-2.54$ & $1.58(2.08)$ & \\
\hline Excluding outlier & & & & $0.60(P=0.017)$ \\
\hline Fellow eye & $-0.01(1.09)$ & $-2.05-2.84$ & $0.73(0.80)$ & \\
\hline Same eye & $-0.96(1.65)$ & $-5.47-2.54$ & $1.33(1.36)$ & \\
\hline Excluding UB & & & & $0.83(P=0.027)$ \\
\hline Fellow eye & $0.04(1.12)$ & $-2.05-2.84$ & $0.75(0.83)$ & \\
\hline Same eye & $-1.20(2.35)$ & $-11.54-2.54$ & $1.58(2.11)$ & \\
\hline \multicolumn{5}{|c|}{$\begin{array}{l}M E \text { mean prediction error, } M A E \text { mean absolute prediction error, } U B \text { ultrasound biometry (A-scan), } P \text { value } \\
\text { using the unpaired two-tailed } t \text {-test }\end{array}$} \\
\hline Study & $\begin{array}{l}\text { Number } \\
\text { (macula off) }\end{array}$ & Biometry method & Same/fellow eye & $\begin{array}{l}\mathrm{MAE} \pm \mathrm{SD} \\
\text { (dioptres) }\end{array}$ \\
\hline \multirow{2}{*}{$\begin{array}{l}\text { El-Khayat et al. } \\
\text { (this study) }\end{array}$} & 42 & $\mathrm{OB}(38)+\mathrm{UB}(4)$ & Fellow eye & $0.73 \pm 0.80$ \\
\hline & 42 & $\mathrm{OB}(38)+\mathrm{UB}(4)$ & Same eye & $1.57 \pm 2.08$ \\
\hline \multirow{3}{*}{$\begin{array}{l}\text { Abou-Shousha et al. } \\
\text { [9] }\end{array}$} & 100 & OB & Same eye & $1.18 \pm 0.84$ \\
\hline & 100 & UB & Same eye & $1.33 \pm 1.06$ \\
\hline & 100 & Vector A/B scan & Same eye & $0.59 \pm 0.48$ \\
\hline \multirow[t]{3}{*}{ Rahman et al. [8] } & 54 & $\mathrm{OB}+\mathrm{UB}$ & $\begin{array}{l}\text { Same }(46)+ \\
\text { Fellow eye }(8)\end{array}$ & $0.73 \pm 0.71$ \\
\hline & 13 & OB & Same eye & $0.65 \pm 0.71$ \\
\hline & 33 & UB & Same eye & $0.77 \pm 0.72$ \\
\hline \multirow[t]{2}{*}{ Kim et al. [11] } & $\begin{array}{l}26 / 38(68 \%) \\
\text { mac off }\end{array}$ & Average UB + OB & Same eye & $0.81 \pm 0.81$ \\
\hline & $\begin{array}{l}14 / 25(56 \%) \\
\text { mac off }\end{array}$ & $\begin{array}{l}\text { Average } \mathrm{UB}+\mathrm{OB} \\
\text { delayed phaco }\end{array}$ & Same eye & $0.48 \pm 0.29$ \\
\hline
\end{tabular}

$O B$ optical biometry, $U B$ ultrasound biometry (A-scan), $S D$ standard deviation
Table 3 Mean absolute prediction error (MAE) of different biometry methods from different studies for phacovitrectomy for macula off retinal detachment biometry methods and in some cases surgeon discretion as to which measurements to select. This makes it difficult to identify the accuracy of individual biometry methods and may introduce bias as only "accurate" measurements were being selected. Nevertheless, our results from fellow-eye biometry compare favourably with these studies, suggesting that, generally, it is a reasonable method to use.

Conditions that may preclude the use of fellow-eye biometry include significant pre-op anisometropia [15], fellow-eye corneal disease (may affect $\mathrm{K}$ readings) [16] and fellow-eye conditions that affect the reliability of axial length measurement, e.g. poor vision (poor fixation) [17], significant media opacity/cataract (poor transmission of light) $[17,18]$ and macular elevation/oedema (underestimation of axial length) [19].

It would be useful to have an objective way to determine the quality of the same-eye AL measurements. This would assist with the challenging decision about which AL measurement method to use. With mac off RRD, several mechanisms, other than poor fixation [9], are thought to contribute to the inaccuracy of OB. Firstly, OB may detect and measure to the detached retina rather than the retinal pigment epithelium, giving a good SNR, but incorrect AL [20]. Secondly, light scatter of the incoming and outgoing rays from the detached retina may degrade scan quality [8]. For routine OB, a SNR of 2 or above is generally regarded as indicative of a good-quality scan [21]. However, due to OB sometimes detecting detached retina with a good SNR, it may not be a useful threshold in RRD. Indeed, all our same-eye OB measurements had a SNR above 2, even though some were clearly inaccurate. Conversely, as mac off RRD may degrade scan quality through light scatter, there may still be a negative correlation between SNR and error in refractive outcomes. We found that SNR had a negative correlation with the error in refractive outcomes from the same-eye biometry. However, this was just short of significance. Further studies with a larger sample size may indicate a significant correlation and may give 
guidance on the level of SNR to accept for same-eye OB for mac off RRD.

Some of the inaccuracies mentioned above for the sameeye OB can be circumvented by visually inspecting the scan graph printout to determine which peak the axial length is being measured from. If a (detached) retinal peak is erroneously picked up, then this can sometimes be manually adjusted to the posterior RPE peak on the IOL Master. Otherwise, the RPE peak from UB can be used with an adjustment of $250 \mu \mathrm{m}$ for normal retinal thickness [22]. These methods may be too complicated for routine use, however, and introduce a degree of subjectivity.

As a 1-mm change in AL corresponds to $\sim 2.7 \mathrm{D}$ refractive error [15], it would be reasonable to assume that for fellow-eye biometry, pre-op anisometropia should correlate with prediction error. We did not find a significant correlation in our study. A larger sample size may reveal a positive correlation.

The strength of this study includes the assessment of a single biometry method for each sample group without user adjustment or selection, indicating reproducibility. The comparisons made are also between identical groups (including surgeon/surgery), controlling for all variables other than the biometry method.

The limitations of this study include its retrospective nature and that the macular status of the detachments was determined only clinically and by visual acuity, and was not confirmed on optical coherence tomography (OCT).

\section{Conclusion}

This retrospective study suggests that, provided it is appropriately used, fellow-eye biometry for phacovitrectomy for mac off RRD leads to refractive outcomes that are accurate and that are better than those from same-eye biometry. It also suggests that for OB in eyes with mac off RRD, there is a negative correlation between SNR and prediction error, although this was not significant. There was no significant correlation between pre-op anisometropia and accuracy of refractive outcomes.

\section{Summary}

\section{What was known before}

- Phacovitrectomy for retinal detachment is a common and successful procedure.

- For macula off rhegmatogenous retinal detachments, axial length measurements can be inaccurate, and there is no consensus on the best biometry method.
- Fellow-eye biometry for lens calculations is an alternative, although there are no published reports on outcomes.

\section{What this study adds}

- This is the only study looking at the accuracy of refractive outcomes from calculations, using fellow-eye biometry for phacovitrectomy for macula off retinal detachments.

- It shows that they are accurate and better than calculations based on the same eye biometry.

\section{Compliance with ethical standards}

Conflict of interest The authors declare that they have no conflict of interest.

Publisher's note: Springer Nature remains neutral with regard to jurisdictional claims in published maps and institutional affiliations.

\section{References}

1. Demetriades A-M, Gottsch JD, Thomsen R, Azab A, Stark WJ, Campochiaro PA, et al. Combined phacoemulsification, intraocular lens implantation, and vitrectomy for eyes with coexisting cataract and vitreoretinal pathology. Am J Ophthalmol. 2003;135:291-6.

2. Panozzo G, Parolini B. Cataracts associated with posterior segment surgery. Ophthalmol Clin. 2004;17:557-68.

3. Jackson TL, Donachie PHJ, Sallam A, Sparrow JM, Johnston RL. United Kingdom national ophthalmology database study of vitreoretinal surgery: report 3, retinal detachment. Ophthalmology. 2014;121:643-8.

4. Jeoung JW, Chung H, Yu HG. Factors influencing refractive outcomes after combined phacoemulsification and pars plana vitrectomy: results of a prospective study. J Cataract Refract Surg. 2007;33:108-14.

5. Rahman R, Kolb S, Bong CX, Stephenson J. Accuracy of useradjusted axial length measurements with optical biometry in eyes having combined phacovitrectomy for macular-off rhegmatogenous retinal detachment. J Cataract Refract Surg. 2016;42:1009-14.

6. Newman DK, Burton RL. Primary vitrectomy for pseudophakic and aphakic retinal detachments. Eye (Lond). 1999;13(Pt 5): 635-9.

7. Manvikar SR, Allen D, Steel DHW. Optical biometry in combined phacovitrectomy. J Cataract Refract Surg. 2009;35:64-9.

8. Rahman R, Bong CX, Stephenson J. Accuracy of intraocular lens power estimation in eyes having phacovitrectomy for rhegmatogenous retinal detachment. Retina. 2014;34:1415-20.

9. Abou-Shousha M, Helaly HA, Osman IM. The accuracy of axial length measurements in cases of macula-off retinal detachment. Can J Ophthalmol. 2016;51:108-12.

10. Pongsachareonnont $P$, Tangjanyatam S. Accuracy of axial length measurements obtained by optical biometry and acoustic biometry in rhegmatogenous retinal detachment: a prospective study. Clin Ophthalmol. 2018;12:973-80. 
11. Kim Y-K, Woo SJ, Hyon JY, Ahn J, Park KH. Refractive outcomes of combined phacovitrectomy and delayed cataract surgery in retinal detachment. Can J Ophthalmol. 2015; 50:360-6.

12. Cartwright NEK, Johnston RL, Jaycock PD, Tole DM, Sparrow JM. The Cataract National Dataset electronic multicentre audit of 55567 operations: when should IOLMaster biometric measurements be rechecked? Eye. 2010;24:894-900.

13. Anon. Optimized IOL constants for the ZEISS IOLMaster. Available at: http://ocusoft.de/ulib/c1.htm. Accessed 29 Aug 2018.

14. Gale RP, Saldana M, Johnston RL, Zuberbuhler B, McKibbin M. Benchmark standards for refractive outcomes after NHS cataract surgery. Eye. 2009;23:149-52.

15. Olsen T. Calculation of intraocular lens power: a review. Acta Ophthalmol Scand. 2007;85:472-85.

16. Lewis JR, Knellinger AE, Mahmoud AM, Mauger TF. Effect of soft contact lenses on optical measurements of axial length and keratometry for biometry in eyes with corneal irregularities. Invest Ophthalmol Vis Sci. 2008;49:3371-8.
17. Rajan MS, Keilhorn I, Bell JA. Partial coherence laser interferometry vs conventional ultrasound biometry in intraocular lens power calculations. Eye (Lond). 2002;16:552-6.

18. Hill W, Angeles R, Otani T. Evaluation of a new IOLMaster algorithm to measure axial length. J Cataract Refract Surg. 2008;34:920-4.

19. Kovács I, Ferencz M, Nemes J, Somfai G, Salacz G, Récsán Z. Intraocular lens power calculation for combined cataract surgery, vitrectomy and peeling of epiretinal membranes for macular oedema. Acta Ophthalmol Scand. 2007;85:88-91.

20. Lege BAM, Haigis W. Laser interference biometry versus ultrasound biometry in certain clinical conditions. Graefes Arch Clin Exp Ophthalmol. 2004;242:8-12.

21. Suto C, Sato C, Shimamura E, Toshida H, Ichikawa K, Hori S. Influence of the signal-to-noise ratio on the accuracy of IOLMaster measurements. J Cataract Refract Surg. 2007;33:2062-6.

22. Steel D. Refractive outcome and possible errors following combined phaco-vitrectomy. In: Lois $\mathrm{N}$, Wong $\mathrm{D}$, editors. Complications of vitreo-retinal surgery. Philadelphia: Wolters Kluwer Health/Lippincott Williams \& Wilkins; 2013. p. 260-5. 